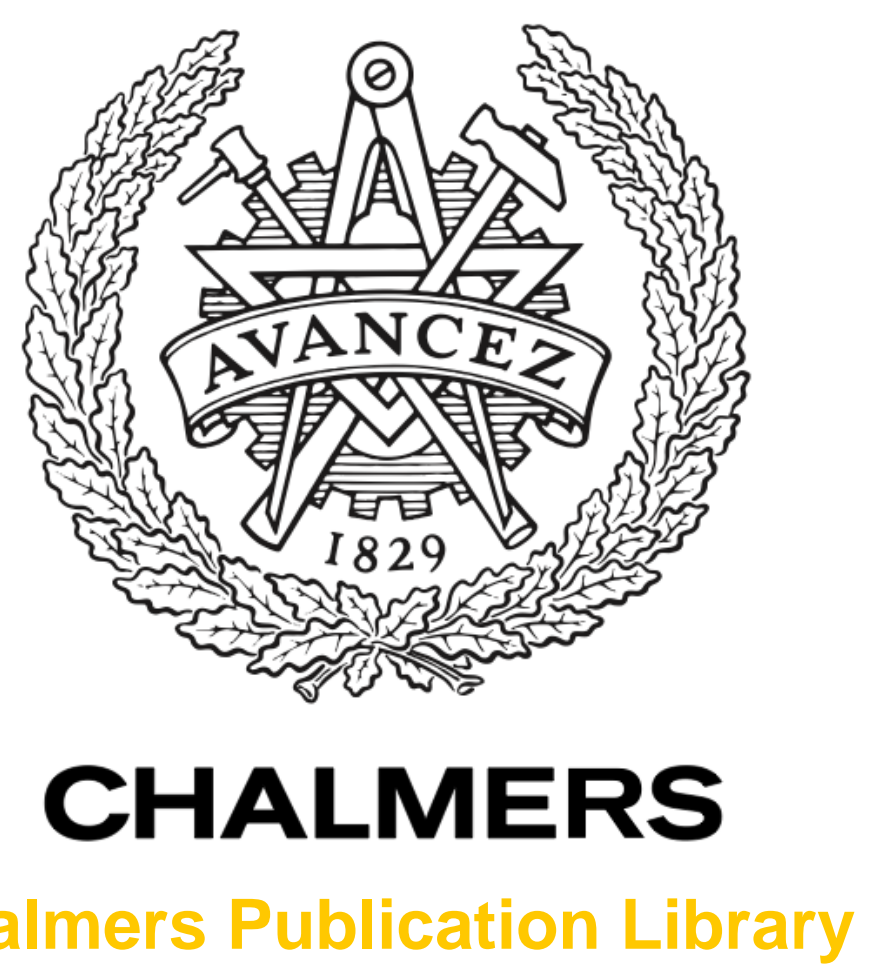

\title{
Experimental Investigation of the Number of Independent Samples and the Measurement Uncertainty in a Reverberation Chamber
}

This document has been downloaded from Chalmers Publication Library (CPL). It is the author's version of a work that was accepted for publication in:

IEEE transactions on electromagnetic compatibility (ISSN: 0018-9375)

Citation for the published paper:

Chen, X. (2013) "Experimental Investigation of the Number of Independent Samples and the Measurement Uncertainty in a Reverberation Chamber". IEEE transactions on electromagnetic compatibility, vol. 55(5), pp. 816-824.

Downloaded from: http://publications.lib.chalmers.se/publication/185176

Notice: Changes introduced as a result of publishing processes such as copy-editing and formatting may not be reflected in this document. For a definitive version of this work, please refer to the published source. Please note that access to the published version might require a subscription. 


\title{
Experimental Investigation of the Number of Independent Samples and the Measurement Uncertainty in a Reverberation Chamber
}

\author{
Xiaoming Chen
}

\begin{abstract}
Due to the complicated and time-varying boundary conditions, reverberation chamber (RC) measurements are usually studied using statistical analysis. Hence, it is of importance to know the number of independent samples of the measurements. Different methods for estimating the number of independent samples has been proposed in the literature. This paper uses the spatial degrees of freedom method to estimate the independent sample number. Comparing with the commonly used autocorrelation function method, the proposed method requires less computation time and provides a smaller estimation bias for correlated samples and a smaller estimation variance once above a certain sample number. The estimated independent sample number can be used to explain the dependences of the measurement uncertainty on frequency, mode stirrers, and RC loading. Finally, the estimated independent sample number is used to predict the standard deviation (STD) of the average power level for different loading configurations, where good agreements are observed.
\end{abstract}

Index Terms-Degrees of freedom (DoF), independent sample number, reverberation chamber (RC), standard deviation (STD).

\section{INTRODUCTION}

$\mathbf{T}$ HE reverberation chamber (RC) has been used for electromagnetic compatibility (EMC) tests [1]-[12] as well as over-the-air (OTA) measurements of active devices [13]-[16]. Due to the complicated and time-varying test conditions, RC measurements are usually studied from a statistical point of view [1]. Therefore, it is of importance to have enough independent samples to ensure an accurate statistical analysis of the measured data. Different methods have been proposed in the literature for estimating the number of independent samples $N_{\text {ind }}$. The most commonly used method for estimating $N_{\text {ind }}$ is the autocorrelation function (ACF) method, see e.g., [2]-[7]. It has been recognized that the ACF method gives only coarse estimations [8]; therefore, modified ACF methods was proposed [2], which inevitably suffers from high-computational complexity.

In this paper, we resort to a simple yet accurate $N_{\text {ind }}$ estimator that has been used in [9] for determining the independent turntable platform positions. As explained in [9], this estimator is

Manuscript received October 17, 2012; revised December 25, 2012; accepted January 21, 2013. Date of publication February 8, 2013; date of current version October 10, 2013

The author is with the Department of Signals and Systems, Chalmers University of Technology, Gothenburg 412 91, Sweden (e-mail: xiaoming. chen@chalmers.se).

Color versions of one or more of the figures in this paper are available online at http://ieeexplore.ieee.org.

Digital Object Identifier 10.1109/TEMC.2013.2242473 rooted from majorization theory (cf., [17, Sec. 4.1.2]), which specifically deals with spatial correlations. It was found out recently that this estimator had been derived in the 1960s [18]. Later on, this estimator was termed spatial degrees of freedom (DoF) in [19] and recently used in [10] for estimating $N_{\text {ind }}$ of the whole stirring sequence.

This paper has three main contributions compared with the previous work (especially [10]). First, the work in [10] used samples in the frequency domain with up to $1 \mathrm{GHz}$ frequency bandwidth for the estimation, which limited the frequency resolution of the estimated $N_{\text {ind }}$. In this paper, we use the divide and conquer approach to separate the whole stirring sequence into different subsets (according to their stirring mechanisms) and estimate $N_{\text {ind }}$ in each subset while treating samples of the other subsets as observations. In this way, we preserve the finest frequency resolution (i.e., the measuring frequency step), which enable us to observe any frequency variation of $N_{\text {ind }}$ estimates. Second, since the DoF estimator suffers from underestimation with finite observations, effort has been exerted in selecting the uncorrelated frequency samples and in choosing better covariance matrix estimation [10]. In this paper, however, it is found out that the underestimation associated with the DoF estimator is negligible for moderately correlated data (e.g., with a uniform complex correlation as small as 0.3 in magnitude). This implies that the underestimation problem can be circumvented or alleviated by oversampling the field in the RC. Third, while almost all the previous $N_{\text {ind }}$ estimation studies were conducted with statistical analyses only, no one has compared the $N_{\text {ind }}$ estimates with the actual measured uncertainty, e.g., standard deviation (STD) of the average power level. In this paper, the estimated $N_{\text {ind }}$ was compared with the measured STD based on extensive measurement campaign in an RC, based on which the DoF estimator (without any estimation correction) is validated qualitatively. It is shown that the RC uncertainty can be estimated using the estimated $N_{\text {ind }}$ based on only one set of measurement instead of many sets independent measurements.

In the following Section II, we introduce the ACF and DoF methods and present a possible way to alleviate the underestimation of the DoF method. In Section III, we compare the estimation performance of the ACF and DoF methods using Monte Carlo simulations. The superiority of the DoF method is clearly demonstrated. In Section IV, we apply the DoF method to measured data in an RC. Based on the extensive measurement campaign in the $\mathrm{RC}$, we are able to explain the measurement uncertainty using the estimated $N_{\text {ind }}$. Section $\mathrm{V}$ concludes this paper. 


\section{ESTIMATOR OF INDEPENDENT SAMPLE NUMBER}

Before presenting the DoF estimator, we briefly introduce the commonly used ACF method for performance comparisons later on. Note that all the equations in this section are related to a single frequency of interest; yet for notational convenience, the frequency dependence is omitted.

\section{A. ACF Method}

The estimated $N_{\text {ind }}$ using the ACF method can be written as

$$
N_{\text {ind }}=\frac{N_{\text {meas }}}{\Delta}
$$

where $N_{\text {meas }}$ is the number of measured samples and $\Delta$ represents the offset at which the autocorrelation coefficient of the measured samples, i.e.,

$$
\operatorname{ACF}(\Delta)=\frac{\sum_{n=0}^{N_{\text {meas }}-\Delta-1} x_{n+\Delta} x_{n}^{*}}{\sum_{n=0}^{N_{\text {meas }}-1} x_{n} x_{n}^{*}}
$$

where the superscript $*$ is the conjugate operator, has dropped to $1 / e \approx 0.37$.

The ACF method is usually applied to the whole dataset. However, when antenna, mode, and platform stirrings are employed in an RC measurement, it is difficult to determine the optimal stirring sequences if the whole dataset is used. A wiser choice would be to apply the ACF method to each of the stirring sequences to determine the number of independent fixed antennas $N_{\text {ind, ant }}$, the number of independent mode-stirring positions $N_{\text {ind,st }}$, and the number of independent platform-stirring positions $N_{\text {ind,pf }}$, respectively. In this case, the obtained offset $\Delta$ is a function of the stirring sequence of interest. For example, if $N_{\text {ind,st }}$ is of interest, then the number of realizations $N$ is the product of the numbers of the other stirring sequences and the corresponding $\Delta$ is a function of mode-stirring position. To improve the estimation accuracy, we take an average of $\Delta$ over all the $N_{\text {st }}$ mode-stirring positions before substituting it to (1). Another way of improving the estimation accuracy is to calculate $N_{\text {ind,st }}$ as a function of the mode-stirring sequence instead and then average $N_{\text {ind,st }}$ over all the $N_{\text {st }}$ mode-stirring positions. One can easily verify by simulations that the latter way (i.e., averaging $N_{\text {ind,st }}$ ) results in slower convergence rate than the former way (i.e., averaging $\Delta$ ). Therefore, we will use the former way for the ACF method throughout this paper.

\section{B. DoF Method}

Instead of estimating $N_{\text {ind }}$ of the whole stirring sequence using frequency samples as observation [10], we divide the whole stirring sequence into three subsets and estimate $N_{\text {ind }}$ of each of the subset while treating the other two subsets as observation (i.e., divide and conquer approach). In this way, we preserve the finest frequency resolution and avoid the potential problem of averaging frequency samples with different statistical properties. Specifically, the number of independent samples in an RC can be decomposed as (cf., Section II-A)

$$
N_{\text {ind }}=N_{\text {ind,ant }} N_{\text {ind, st }} N_{\text {ind,pf }} .
$$

Note that (3) holds when the three stirring sequences are independent. When there are correlations between the different stirring sequences the right-hand side of (3) serves an upper bound of $N_{\text {ind }}$. Nevertheless, due to the different physical mechanisms of the three stirring sequences, (3) holds in practice, as can be seen in Section IV. Each term at the right-hand side of (3) $N_{\text {ind,l }}$ ( $l$ represents ant, st, or pf) can be estimated using the following procedure [9].

1) Denote the complex field samples at the $m$ th antenna $(l=$ ant), mode-stirring position $(l=\mathrm{st})$, or platform-stirring position $(l=\mathrm{pf})$ as a column vector $\mathbf{x}_{m}, m=1 \ldots N_{l}$.

2) Concatenate $\mathbf{x}_{m}$ into a matrix $\mathbf{X}=\left[\begin{array}{lll}\mathbf{x}_{1} & \ldots & \mathbf{x}_{N_{l}}\end{array}\right]$.

3) Estimate the correlation matrix of $\mathbf{X}$ as

$$
\mathbf{R}=\mathbf{X X}^{H} \text {. }
$$

4) The independent antenna $(l=\mathrm{ant})$, mode-stirrer $(l=\mathrm{st})$, or platform $(l=\mathrm{pf})$ sample number can be estimated as [10], [17]-[19]

$$
N_{\text {ind }, l}=\frac{\operatorname{tr}(\mathbf{R})^{2}}{\operatorname{tr}\left(\mathbf{R}^{2}\right)}=\frac{\left(\sum_{i} \lambda_{i}\right)^{2}}{\sum_{i} \lambda_{i}^{2}}
$$

where the superscript ${ }^{H}$ denotes conjugate transpose, tr represents the trace operator, and $\lambda_{i}$ represents the $i$ th eigenvalue of $\mathbf{R}$. It determines the dimensionality of the column space of $\mathbf{X}$ [20] and therefore the corresponding independent sample number. Because of (5), the proposed method for estimating $N_{\text {ind }}$ is referred to as the DoF method [19]. The accuracy of this method depends on the number for realizations. For large realization numbers, (5) and therefore (3) approach asymptotically to their corresponding true values, respectively. Apart from the original derivation in [18], (5) can also be derived using the information theory technique [10]. Note that electromagnetic functions are continuous physical function defined in a norm space with inner product well defined whereas signals can be artificial and usually they are not defined in well-defined spaces. An excellent discussion regarding this point can be found in [11], where the correlation matrix was related to independent positions of the stirrer and physical proprieties are also investigated with a different approach.

Note that although $\mathbf{R}$ in (4) is the maximum likelihood estimation of the true correlation matrix $\mathbf{R}_{0}$, the eigenvalue of $\mathbf{R}$, i.e., $\lambda_{i}$, are biased estimates of the true eigenvalues [21]. Especially, when the number of realizations is comparable in magnitude to the number of stirring sequence $N_{l}$, estimates of small eigenvalues are biased down, while estimates of large eigenvalues are biased up. Therefore, the estimated eigenvalue vector majorizes the true eigenvalue vector [17] and consequently $\mathbf{R}$ is more correlated than $\mathbf{R}_{0}$. This results in underestimation of $N_{\text {ind,1 }}$ for data with little correlations. To overcome this problem, one can use the improved eigenvalue estimator [21]

$$
\lambda_{i}^{\mathrm{imp}}=N_{l}\left(\lambda_{i}-\mu_{i}\right)
$$

with $\mu_{i}$ as the solutions of

$$
\sum_{i=1}^{N} \frac{\lambda_{i}}{\lambda_{i}-\mu_{i}}=N_{l}
$$


Nevertheless, as will be shown in the next section, the improved eigenvalue estimator (6) is only useful for uncorrelated data and that it is not needed in practice for oversampled (correlated) data, which is actually required for estimating $N_{\text {ind }}$ [4].

Comparing the DoF method with the ACF method, it can be seen that the latter requires more computation because of the $\mathrm{ACF}(2)$ and the inverse mapping needed to determine the offset $\Delta$. Note that the accuracies of both methods depend also on the correlations of the measured data, which has been overlooked in the previous studies. Also note that we assume the data to be drawn from a zero mean random process so that the correlation in both methods is equivalent to covariance. The zero mean random process assumption holds in practice for an RC equipped with platform stirring (cf., [22]). For RCs with nonnegligible K-factors, e.g., [23], [24], both methods can be extended to the covariance case by removing the mean value in a straightforward manner. In the next section, we will compare the performance of the DoF method with that of the ACF method and study their dependences on the number of realizations and correlations of measured data via simulations.

\section{SIMULATION}

We resort to simulations to study the performance of the DoF method and compare it with the ACF method. For the sake of easy exposition and without loss of generality, we focus on the estimating $N_{\text {ind,st }}$ (estimations of the independent sample numbers for the other two stirring sequences will be studied in the next section with measured data). We first numerically generated an $N_{\text {st }} \times N$ random matrix $\mathbf{X}_{w}$ consisting independent and identically distributed (i.i.d.) Gaussian elements. Given the true correlation matrix $\mathbf{R}_{0}$, the correlations can be introduced to the mode-stirring positions by [9]

$$
\mathbf{X}=\mathbf{R}_{0}^{1 / 2} \mathbf{X}_{w}
$$

where the superscript ${ }^{1 / 2}$ can be implemented as, e.g., the Cholesky decomposition [20].

Note that due to finite realizations, the estimate $\mathbf{R}=\mathbf{X} \mathbf{X}^{H}$ differs from $\mathbf{R}_{0}$ almost surely, but as $N$ increases the estimated $N_{\text {ind,st }}$ approaches its true value. This is illustrated in Fig. 1 for uniform correlations with four correlation coefficients, i.e., $\rho=$ $0,0.3,0.5,1$, by applying the DoF method to the correlated data $\mathbf{X}$ (where $N_{\text {st }}$ is assumed to be 10). The solid curves in Fig. 1 represents the estimated $N_{\text {ind,st }}$ using (5) directly and the dotted curves represents the estimated $N_{\text {ind,st }}$ using the improved eigenvalue estimates (6) [i.e., by replacing the eigenvalue estimates in (5) with the improved eigenvalue estimates (6)]. For $\rho=0$, the mode-stirring positions are independent (to be exact, the random fields are uncorrelated, and since the field in an $\mathrm{RC}$ is Gaussian distributed [1], they are independent as well); therefore, the asymptotic $N_{\text {ind,st }}=10$; for $\rho=1$ all the modestirring positions are totally dependent, therefore the asymptotic $N_{\text {ind,pf }}=1$. As can be seen from Fig. 1 , the estimated $N_{\text {ind,st }}$ converges to its true value as $N$ increases and that the $N_{\text {ind,st }}$ underestimation for the uncorrelated data can be partially corrected by using the improved eigenvalue estimates (6) and that the underestimation (and therefore the estimation error) is more

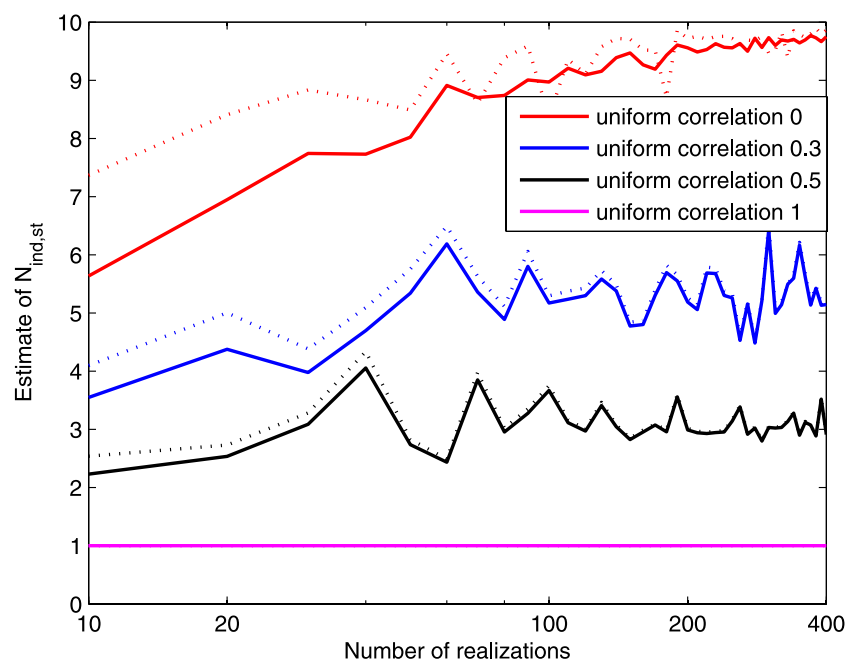

Fig. 1. Estimated $N_{\text {ind st }}$ using the DoF method as a function of number of realizations with $N_{\mathrm{st}}=10$. Different colors represent different correlation cases. The solid curves represents the estimated $N_{\text {ind,st }}$ using (5) directly and the dotted curves represents the estimated $N_{\text {ind,st }}$ using the improved eigenvalue estimates (6). For the case of total correlation, the solid and dotted curves overlap.

severer for less correlated samples. As the sample correlation increases, the performance of the DoF method improves, i.e., the estimates converge faster to their corresponding true values.

Since estimating the number of independent samples requires oversampling the field (cf., [4]), in practice the measured data are correlated. It is known from Fig. 1 that with more correlated data, there is no need to use the improved eigenvalue estimator (6), which has four times longer computational time according to simulations. This finding is significant in that employing any of the improved estimators will drastically increase the computational complexity. As a result, we will use (5) directly for the DoF method hereafter.

We next run simulations to compare the estimation performance of the DoF method with that of the ACF method, assuming ten mode-stirring positions, i.e., $N_{\text {st }}=10$. We first compare the biasness of the two methods by plotting the estimated $N_{\text {ind,st }}$ as a function of number of realizations with uniform correlations, as shown in Fig. 2. It can be seen that 1) the DoF method (solid curves) tends to underestimate $N_{\text {ind,st }} ; 2$ ) the ACF method has larger estimation bias than the DoF method; and 3 ) estimates of the two methods tend to converge to the true value as the number of realizations increases and, for correlated samples, the convergence rate of the DoF method is much faster than that of the ACF method. Note that the underestimation of the DoF method for uncorrelated data in Fig. 2 is more severe than that in Fig. 1. This is because that the majorization problem (cf., Section II-B) is more profound when the number of realization per stirring position (i.e., $N / N_{\text {st }}$ in this case) is smaller. As explained before, since only the maximum $N_{\text {ind }}$ is of interest, in practice the measured data for determining $N_{\text {ind }}$ are oversampled, which favors the DoF method even more.

To study the variance of the two methods, we then repeat the simulation 1000 times to calculated the STD of the estimates of $N_{\text {ind,st }}$. For a realistic comparison, an estimated correlation 


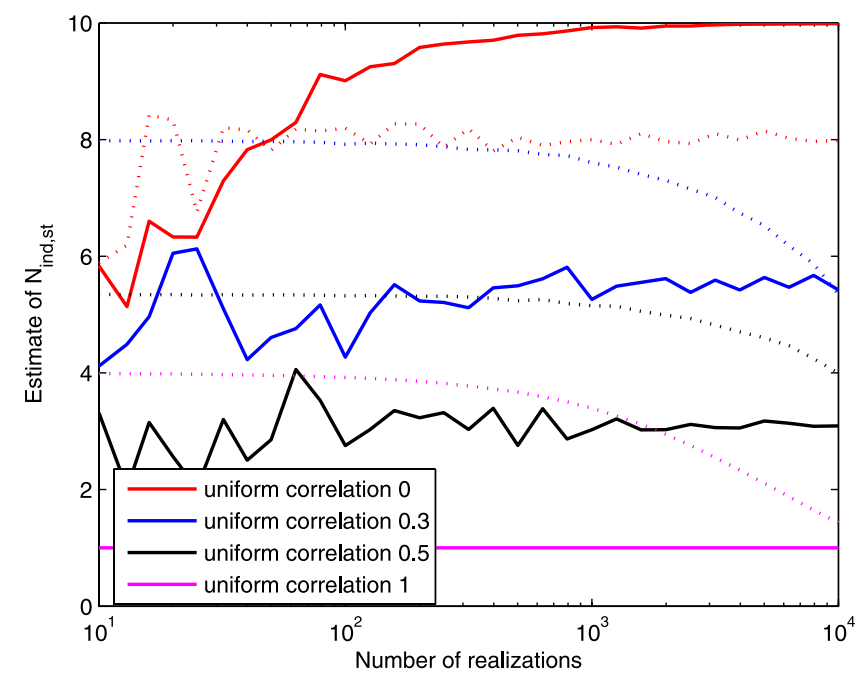

Fig. 2. Estimated $N_{\text {ind,st }}$ using the DoF and ACF methods as a function of number of realizations with $N_{\mathrm{st}}=10$. Different colors represent different correlation cases. The solid curves represents the estimated $N_{\text {ind,st }}$ using the DoF method (5) and the dotted curves represents the estimated $N_{\text {ind,st }}$ using the ACF method.

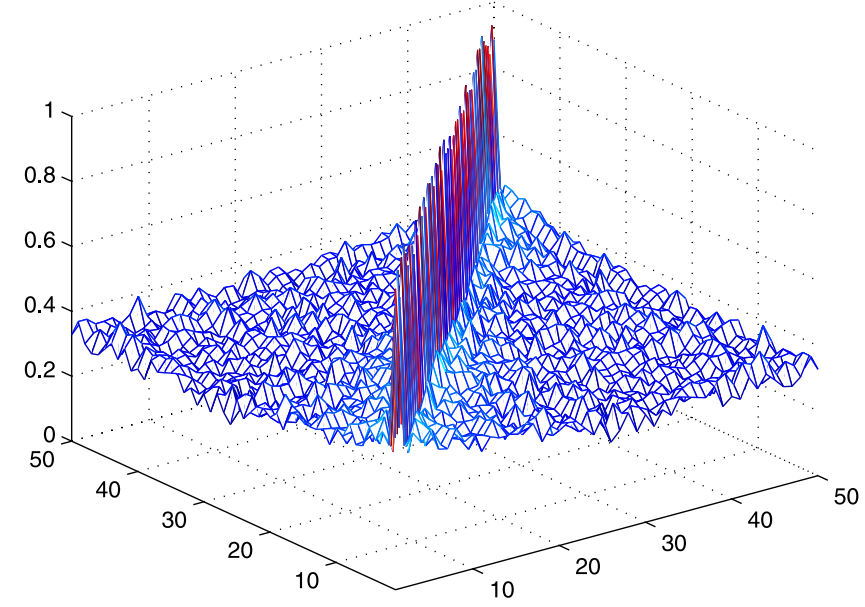

Fig. 3. Magnitudes of correlation coefficients between measured samples at different mode-stirring positions in an RC.

matrix from the measured samples at 50 mode-stirring positions in an RC (cf., Section IV) is used as $\mathbf{R}_{0}$ for this simulation. For simplicity, we refer to this estimated correlation matrix as measured correlation matrix. The measured correlation matrix is shown in Fig. 3, from which it can be seen that the measured data are correlated (because of oversampling). To reduce the computation burden and focus on practical sample numbers, we confine the number of realizations between 50 and 200. The calculated STDs of the estimates of both methods are shown in Fig. 4. As can been seen, the accuracy of the DoF method depends on the number of realization $N$ and that it has a smaller variance than the ACF method when $N$ is larger than 85. (This sample number is not difficult to obtain for practical $\mathrm{RC}$ measurements.) Moreover, based on the observation of the simulations, the computation time of the ACF method for one realization is larger than that of the DoF method by a factor of

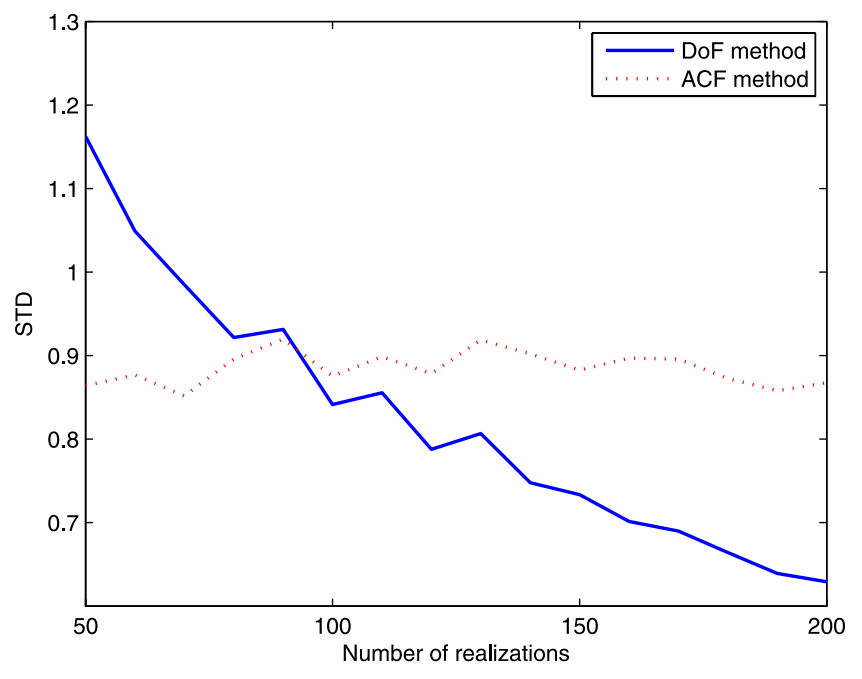

Fig. 4. STD of the estimated $N_{\text {ind,st }}$ using DoF and ACF methods with the measured correlation matrix shown in Fig. 2.

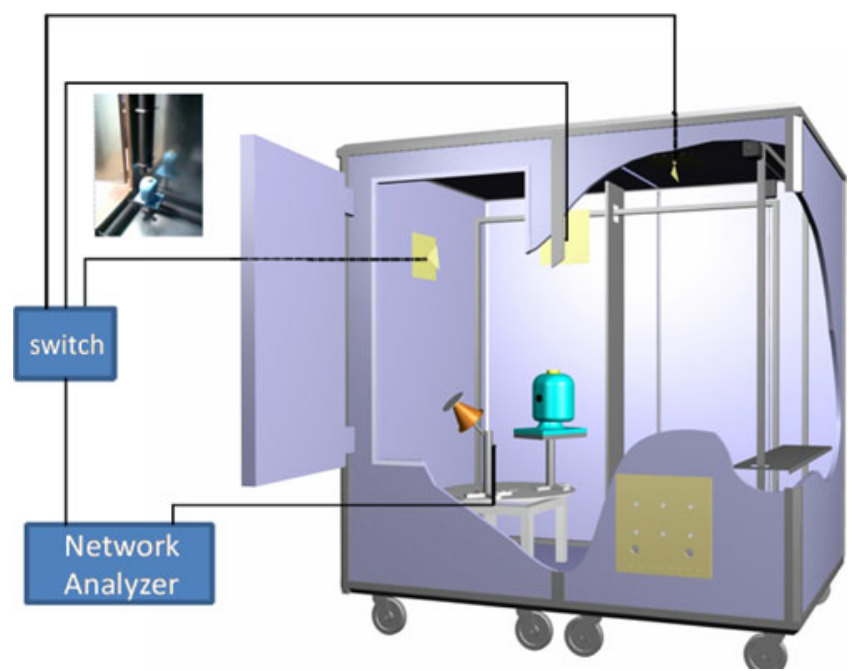

Fig. 5. Drawing of Bluetest RC with two mechanical plate stirrers, one platform and three wall antennas (The inserted little photo in the upper left corner shows the head phantom and the location of the three absorber-filled PVC cylinders of load2 configuration.

10 and that this factor increases linearly with increasing number of realizations for averaging (cf., Section II-A).

\section{MeAsurement}

Measurements were performed from 500 to $3000 \mathrm{MHz}$ in the Bluetest HP RC [16] that has a size of $1.80 \times 1.75 \times$ $1.25 \mathrm{~m}^{3}$ (a drawing of which is shown in Fig. 5). This frequency range is chosen because it covers most of the spectrums of telecommunications for active OTA measurements, e.g., [13]. The RC has two mode-stirring plates, a turn-table platform (on which a wideband discone antenna is mounted), and three antennas mounted on three orthogonal walls (referred to as wall antennas hereafter). The wall antennas are actually wideband half-bow-tie antennas. During the measurement, the turn-table platform was step-wisely moved to 20 platform stirring positions evenly distributed over one complete platform rotation; at 


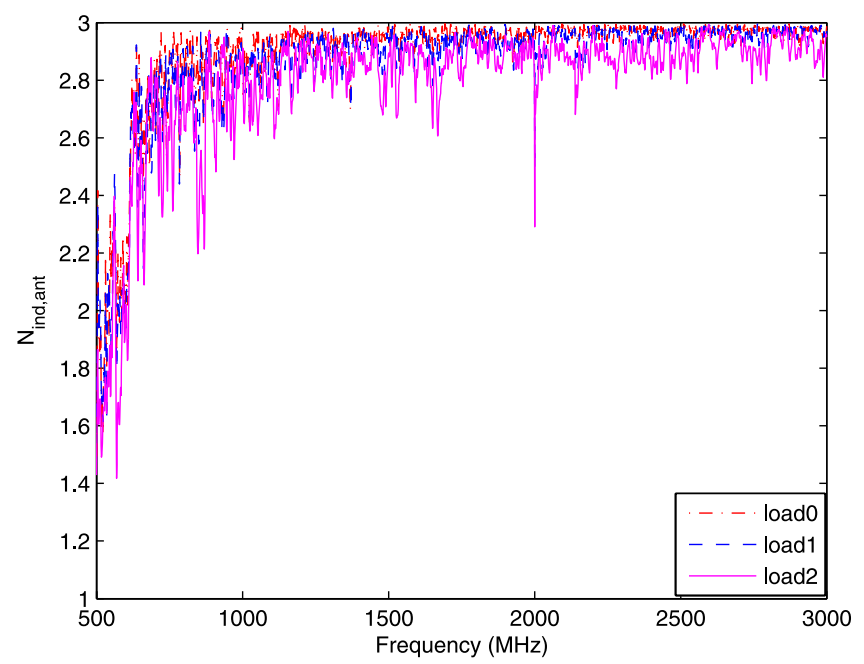

Fig. 6. Estimated $N_{\text {ind, ant }}$ using the DoF method based on measurement in the RC.

each platform-stirring position the two plates were simultaneously and step-wisely moved to 50 positions (equally spanned on the total distances that they can travel along two RC walls). At each stirrer position and for each wall antenna a full frequency sweep was performed by a vector network analyzer (VNA) with a frequency step of $1 \mathrm{MHz}$, during which the scattering parameter (S-parameter) is sampled as a function of frequency and stirring position. Hence, for this measurement setup, we have three (fixed) wall antennas, 50 plate-stirring positions, and 20 platform-stirring positions, i.e., $N_{\text {ant }}=3, N_{\text {st }}=50$, and $N_{\text {pf }}=20$.

To be able to estimate the measurement uncertainty (in terms of STD of the average power level) and to see the effects $N_{\text {ind,ant }}, N_{\text {ind,st }}$, and $N_{\text {ind,pf }}$ on the measurement uncertainty, the same measurement sequence is repeated nine times, each time with a different height/orientation of the antenna on the platform, i.e., the antenna on the platform was placed with three different heights and at each height it is placed with three different orientations. This nine-measurement procedure was proposed in [16] for uncertainty assessment.

The nine-measurement procedure were repeated for three loading conditions: load 0 (unloaded RC), load 1 (head phantom that is equivalent to a human head in terms of microwave absorption), and load2 (the head phantom plus three Polyvinyl Chloride (PVC) cylinders filled with electromagnetic absorbers cut in small pieces), whose quality factor (Q-factor) is around $1000,550,300$ at $1.5 \mathrm{GHz}$ (see Fig. 3 in [12] for the corresponding Q-factor as a function of frequency). Hereafter measured data from these different loading configurations are simply referred to as load 0 , load1, or load 2 data.

The estimated $N_{\text {ind,ant }}, N_{\text {ind,st }}$, and $N_{\text {ind,pf }}$ are shown in Figs. 6-8, respectively. Since the three wall antennas are sufficiently away from each other and with orthogonal polarizations, it is natural that $N_{\text {ind,ant }}$ is close to 3 for most of the frequencies (cf., Fig. 7). Note that the drop of $N_{\text {ind,ant }}$ below $700 \mathrm{MHz}$ is probably due to the fact that the $\mathrm{RC}$ is undermoded below $700 \mathrm{MHz}$, i.e., the fundamental mode resonance

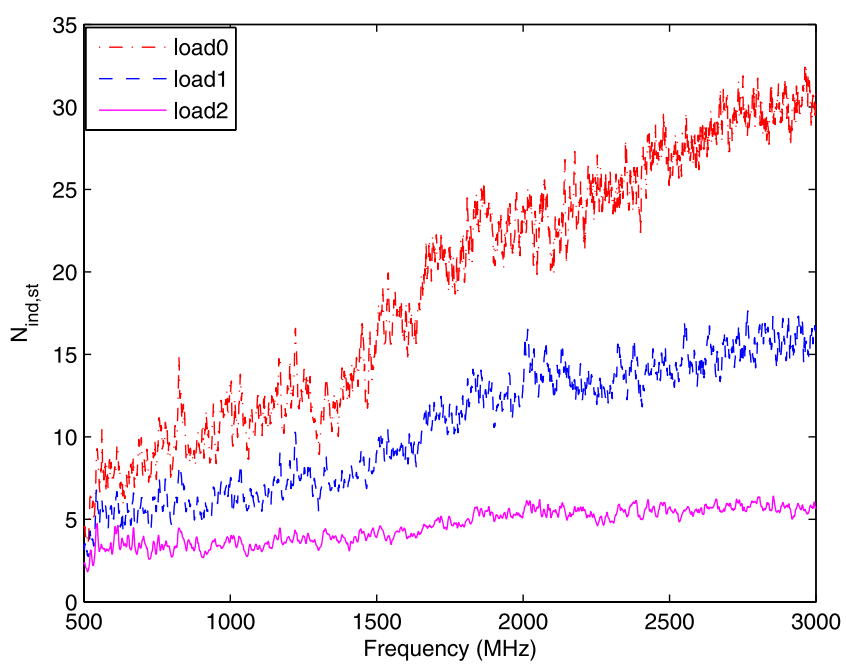

Fig. 7. Estimated $N_{\text {ind,st }}$ using the DoF method based on measurement in the RC.

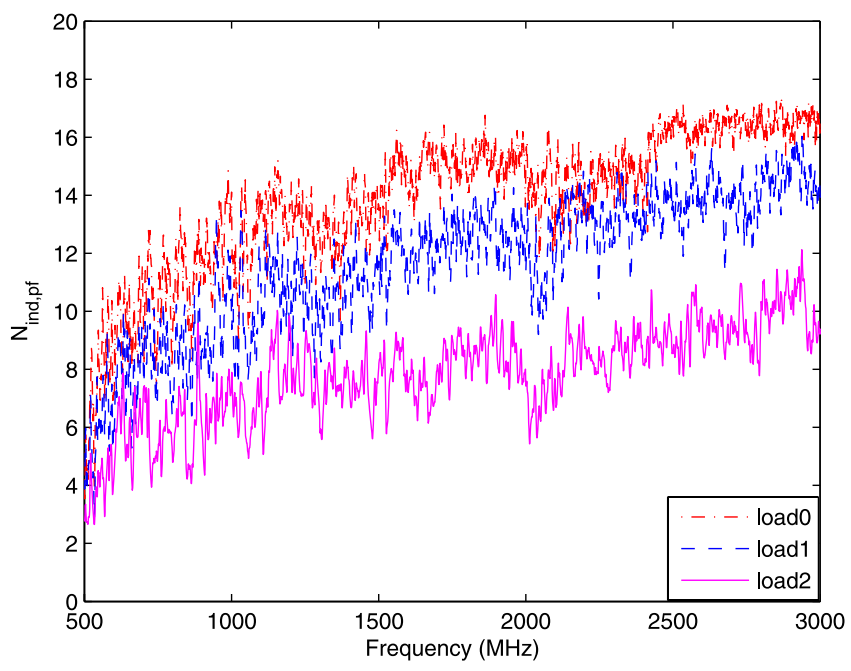

Fig. 8. Estimated $N_{\text {ind,pf }}$ the DoF method based on measurement in the RC.

frequency for the RC in use is $f_{0}=119 \mathrm{MHz}$, giving a lowest usable frequency of about $6 f_{0}=717 \mathrm{MHz}$ (see [25] and reference therein). It can also be seen from Fig. 5 that $N_{\text {ind,ant }}$ is almost independent of the RC loading. This is because that the wall antennas are uncorrelated, i.e., there is no oversampling w.r.t. the antenna stirring. This implies that the measurement uncertainty can be improved by introducing more wall antennas. Nevertheless, since the wall antennas are connected to the VNA via a radio frequency (RF) switch (see Fig. 5), the improved measurement uncertainty by increasing wall antennas will be at the cost of increasing measurement time and hardware expanses (e.g., more antennas, more RF cables, and an RF switch with more ports).

Due to the oversampling of the mode and platform stirrings, the resulting $N_{\text {ind,st }}$ and $N_{\text {ind,pf }}$ depend on the RC loading (see Figs. 7 and 8). As can be seen that $N_{\text {ind,st }}$ and $N_{\text {ind,pf }}$ increase with increasing frequency and decrease with increasing loading. This is rather intuitive: at higher frequencies, the modeand platform-stirring positions are less correlated due to the 
increasing electrical distances; by increasing the RC loading, the angular spread reduces (i.e., the field is less isotropic) and therefore the correlation length [26] (or coherence distance) that is inversely proportional to the angular spread [27] increases, rending more correlated mode- and platform-stirring positions. Note that $N_{\text {ind,st }}$ and $N_{\text {ind,pf }}$ are strictly smaller than $N_{\text {st }}=50$ and $N_{\mathrm{pf}}=20$, respectively, over the whole selected frequency range.

In order to see the impact of $N_{\text {ind }}$ on the measurement uncertainty and verify the DoF method by measurements, we calculated the STD of the average power over the nine-measurements for each loading. For the conciseness of this paper, we will focus on the cases of load 0 and load2. For clear exhibitions, the calculated STD $\sigma$ is plotted using the following dB-transformation [16]:

$$
\sigma_{\mathrm{dB}}=5 \log _{10} \frac{(1+\sigma)}{(1-\sigma)} .
$$

The measured STDs with $N_{\text {pf }}=20, N_{\text {st }}=50$, and $N_{\text {ant }}=\{1,2,3\}$ (i.e., with 1, 2, or 3 wall antennas) are plotted in Fig. 9. The measured STDs with $N_{\text {ant }}=3, N_{\text {pf }}=20$, and $N_{\mathrm{st}}=\{5,10,25,50\}$ are plotted in Fig. 10. Note that the mode-stirring positions are chosen as far away from each other as possible for $N_{\mathrm{st}}=\{5,10,25\}$, e.g., for $N_{\mathrm{st}}=10$, the mode-stirring positions are chosen with a step of 5 out of the total $N_{\mathrm{st}}=50$ positions. The measured STDs with $N_{\text {ant }}=3$, $N_{\text {st }}=50$, and $N_{\text {pf }}=\{1,2,4,10,20\}$ are shown in Fig. 11 . Note that the platform-stirring positions are chosen as far away from each other as possible in a similar manner as the selection of the mode-stirring positions described earlier. Also note that, in order to increase the readability of the plotted STD curves, a 50-MHz smoothing window [16] is applied to the calculated STDs before plotting.

Comparing Figs. 6 and 9, it can be seen that the measurement uncertainty improves with increasing $N_{\text {ant }}$ from 1 to 3 , while $N_{\text {ind, ant }}$ is close to 3 for most of the frequencies, regardless of the RC loading. Comparing Figs. 7 and 10, it can be seen that, for the load 0 case, $N_{\text {ind,st }}$ exceeds 25 above $2 \mathrm{GHz}$, while increasing $N_{\text {st }}$ from 25 to 50 shows clear improvement of the measurement uncertainty above $2 \mathrm{GHz}$ [see Fig. 10(a)]; it can also be seen that, for the load2 case, increasing $N_{\text {st }}$ more than 5 only results in negligible improvements the measurement uncertainty [see Fig. 10(b)], while $N_{\text {ind,st }}$ is only slightly larger than 5 at its largest value. Comparing Figs. 8 and 11, it can be seen that, for the load 0 case, $N_{\text {ind,pf }}$ is larger than 10 for most of the frequencies, while it is beneficial to use more than ten platform-stirring positions [see Fig. 11(a)]; it can also be seen that, for the load 2 case, there is little improvement of the measurement uncertainty by increasing $N_{\mathrm{pf}}$ from 10 to 20 [see Fig. 11(b)], while the largest $N_{\text {ind,pf }}$ value is around 10 for load2. In summary, the experimental observation agrees with the fact that the measurement uncertainty improves with increasing independent samples number. Therefore, the DoF method for estimating the number of independent samples is qualitatively verified by measurements.

To further verify the DoF method, we substitute the estimated $N_{\text {ind,ant }}, N_{\text {ind,st }}$, and $N_{\text {ind,pf }}$ into (3) to obtain the estimated

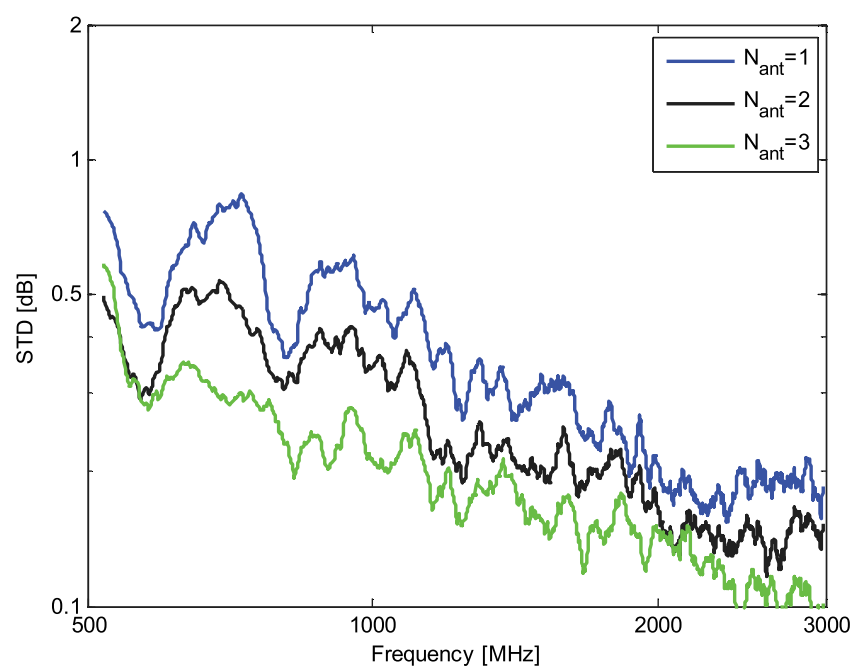

(a)

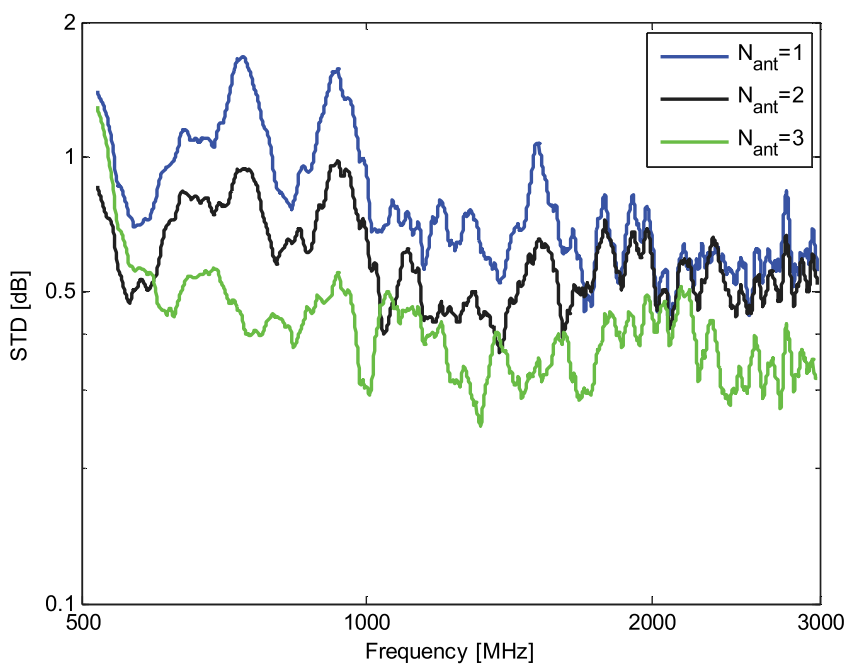

(b)

Fig. 9. Estimated STD of average power over the nine-measurements with different wall antenna numbers for measured data of (a) load0 and (b) load2.

total number of independent samples $N_{\text {ind }}$. The theoretical STD based on the estimated $N_{\text {ind }}$ is

$$
\sigma_{\mathrm{th}}=\frac{1}{\sqrt{N_{\text {ind }}}} .
$$

Applying the dB-transformation (9) to $\sigma_{t h}$, the resulting $\mathrm{dB}$-valued theoretical STD is shown together with the measured STD with all the stirring sequences, i.e., $N_{\text {ant }}=3, N_{\text {st }}=50$, and $N_{\mathrm{pf}}=20$, in Fig. 12. As can be seen, except for the load 2 case, the theoretical STDs based on the DoF method agree well with the estimated STDs based on measurements. The small discrepancy for the load 2 case is reasonable because the field is more spatially correlated for such heavy loading, rendering less independent nine measurements (that were described earlier in this section) for the uncertainty assessment. In other words, the measured STD for load 2 is less accurate than the other two loading cases. Since the theoretical STD based on the estimated $N_{\text {ind }}$ using the DoF method is not affected by the independence of the nine measurements, it is believed that the estimated STD 


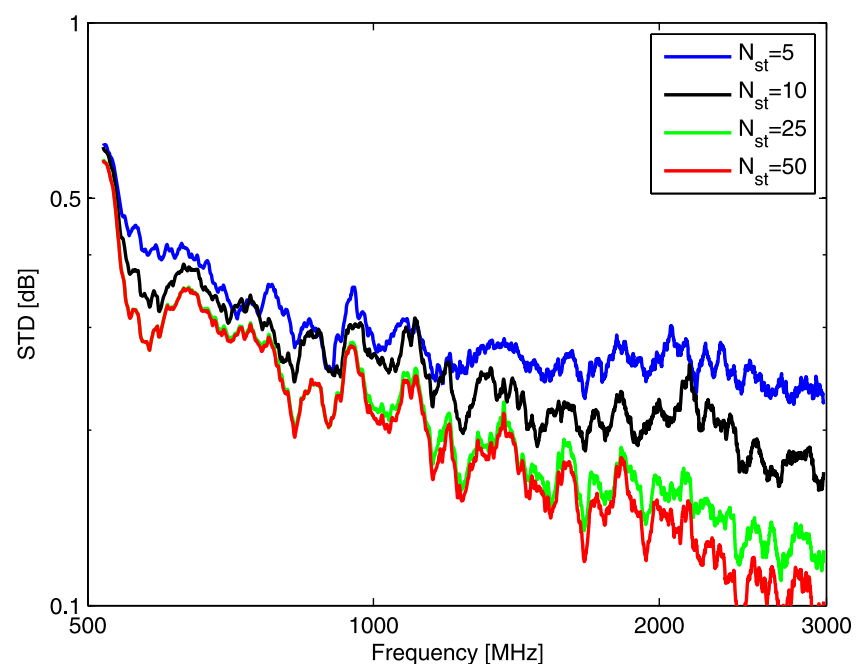

(a)

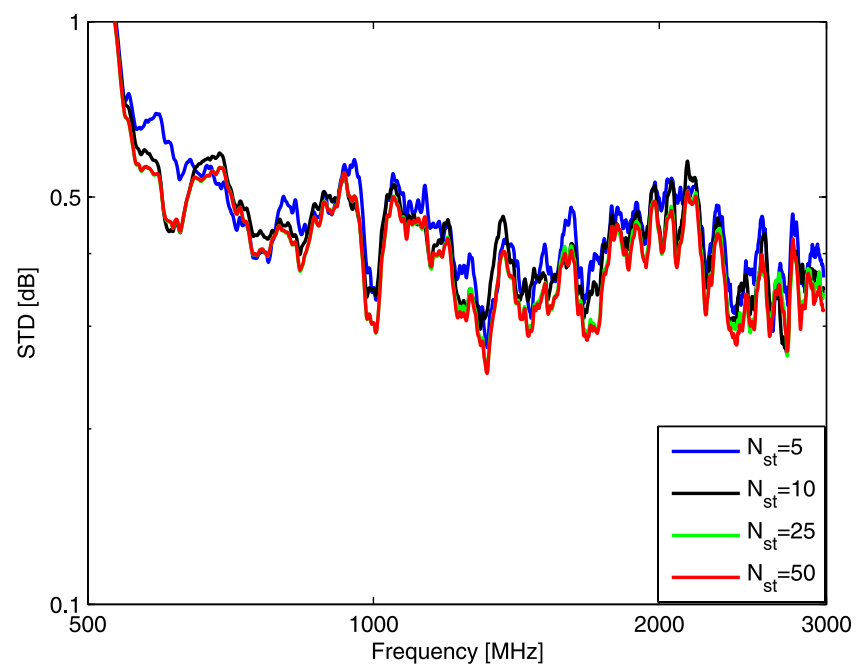

(b)

Fig. 10. Estimated STD of average power over the nine-measurements with different numbers of mode-stirring positions for measured data of (a) load 0 and (b) load2.

by (10) using the DoF method is more accurate for the load2 case.

Note that the good agreements between the theoretical STD (10) using the DoF method and the measured STD is not in contradiction with the findings in [16], where it is claimed that the K-factor (that increases with increasing RC loading [23]) represents a residual error for the measurement uncertainty of an $\mathrm{RC}$, because, unlike the uncertainty model in [16], the estimated $N_{\text {ind }}$ using the DoF method has taken the effect of the RC loading into account (see Figs. 6 and 7).

Nevertheless, since $N_{\text {ind }}$ can be estimated using the DoF method based on only one set of measurement instead of nine, the finding shows that the DoF method can actually be used to predict the measurement uncertainty with much less measurement effort. To the best knowledge of the author, this finding has not been shown in any of the previous work.

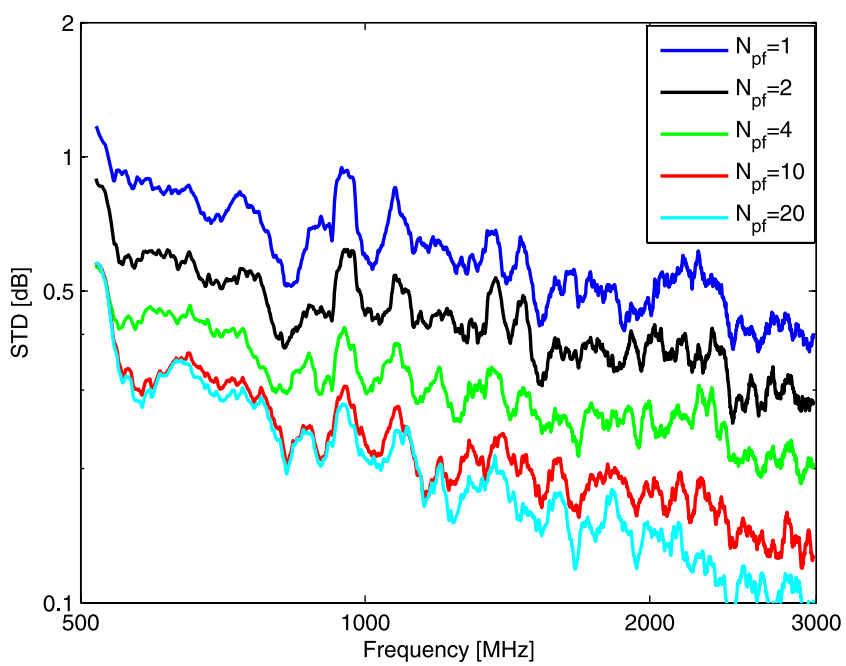

(a)

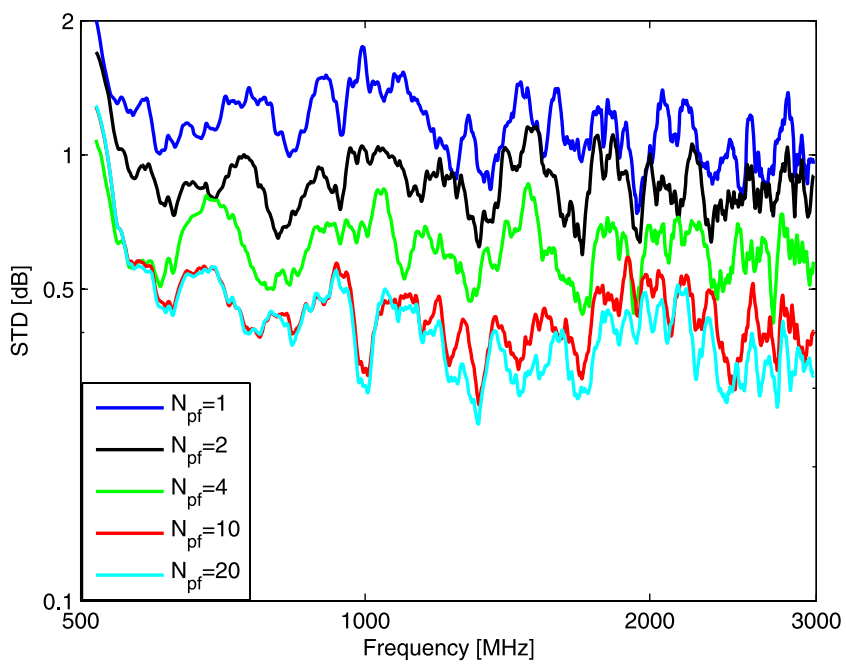

(b)

Fig. 11. Estimated STD of average power over the nine-measurements with different numbers of platform-stirring positions for measured data of (a) load 0 and (b) load2.

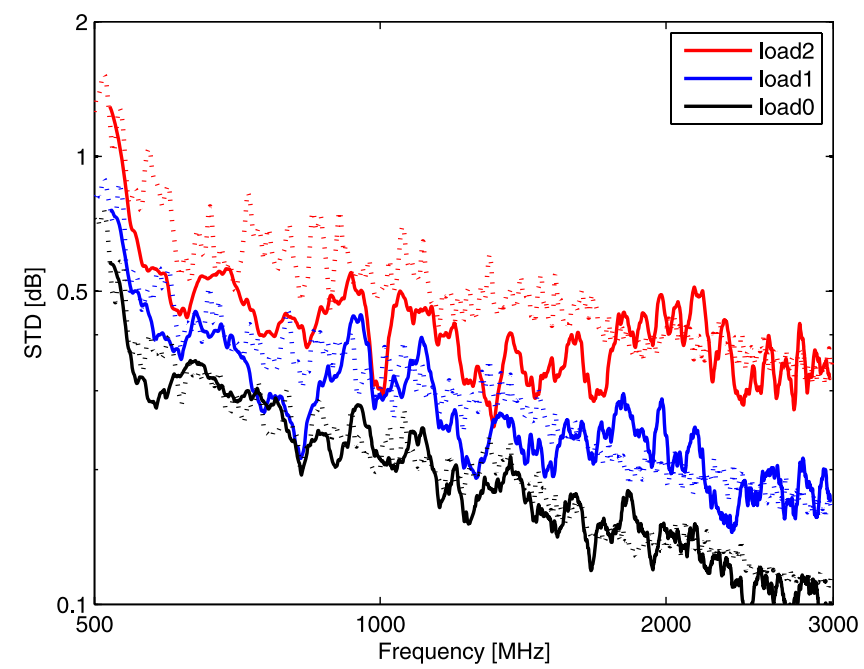

Fig. 12. Comparisons of theoretical STDs based on the estimated $N_{\text {ind }}$ using the DoF method (dotted curves) with the measured STDs (solid curves). 


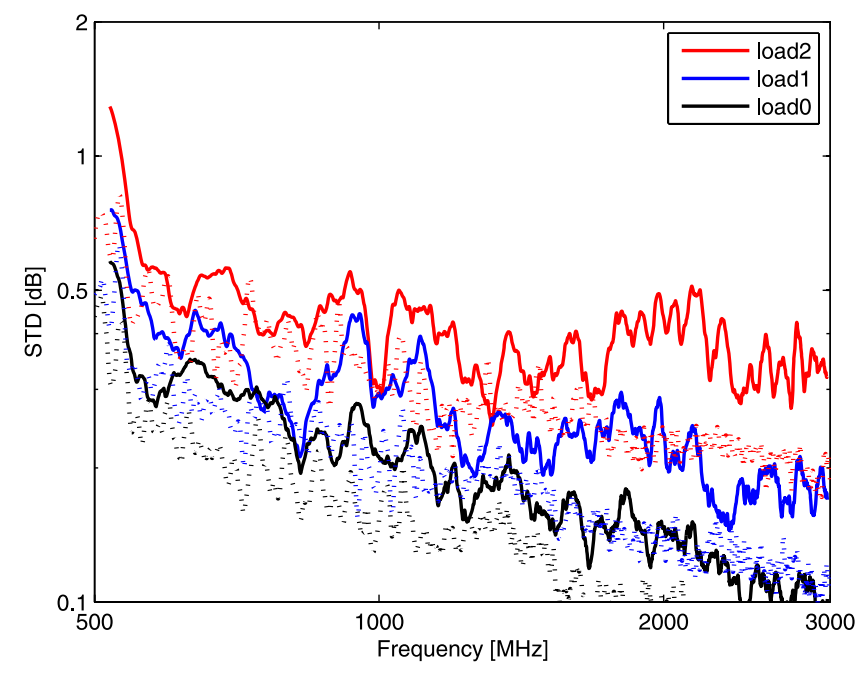

Fig. 13. Comparisons of theoretical STDs based on the estimated $N_{\text {ind }}$ using the ACF method (dotted curves) with the measured STDs (solid curves).

Finally, in order to show the superiority of the DoF method to the ACF one, we apply the ACF method to the same measurement data (in a similar way as the DoF method) and estimate $N_{\text {ind }}$. Fig. 13 shows the comparisons of the theoretical STDs based on (10) and the ACF estimates and the measured STDs using the nine-measurement uncertainty procedure. It is shown clearly that the ACF method results in a poor prediction of the STD (and equivalently poor $N_{\text {ind }}$ estimation).

\section{CONCLUSION}

In this paper, the DoF method is applied to estimate the number of independent samples in an RC. Unlike the work in [10], we use the divide and conquer approach to estimate $N_{\text {ind }}$ without sacrifice the frequency resolution. In addition, we avoid the potential problem of averaging frequency samples (over up to $1 \mathrm{GHz}$ bandwidth [10]) with different statistical properties. The estimation performance of the DoF method was compared with that of the commonly used ACF method. It is found that the estimation bias of the DoF method is smaller than that of the ACF method for correlated samples and that its estimation variance decreases with increasing sample number and is smaller than that of the ACF method when the number of realizations is larger than a certain number (that is easy to obtain for practical RC measurements). The computation time required by the $\mathrm{ACF}$ method is at least 10 times larger than that required by the DoF method. Moreover, it is found that the underestimation problem of the DoF method can be easily and effectively alleviated by oversampling (which is a common practice in determining $N_{\text {ind }}$ [4]). Applying the DoF method to the measured data, the independent sample numbers for different stirring sequences (e.g., mode- and source-stirrings) can be readily determined. This is extremely helpful in choosing the optimal stirring sequences, (i.e., stirring sequences without oversampling yet contain all the necessary information to keep a minimum measurement uncertainty), which enables a reduction of the measurement time without sacrifice of the measurement uncertainty. Finally, we use the nine-measurement uncertainty procedure proposed in [16] to determine the measurement uncertainty. It is shown that the estimated independent sample numbers can be used to well explain the measured STDs with different stirring sequences. The DoF method was further verified by comparing the square root of the estimated independent sample number with the measured STDs, where good agreements are observed. This implies that one can predict the RC STD based on one set of measurements instead of nine using the DoF method, which significantly reduces the measurement effort in RC uncertainty assessment.

\section{REFERENCES}

[1] J. G. Kostas and B. Boverie, "Statistical model for a mode-stirred chamber," IEEE Trans. Electromagn. Compat., vol. 33, no. 4, pp. 366-370, Nov. 1991.

[2] N. Wellander, O. Lunden, and M. Bäckström, "Experimental investigation and mathematical modeling of design parameters for efficient stirrers in mode-stirred reverberation chambers," IEEE Trans. Electromagn. Compat., vol. 49, no. 1, pp. 94-103, Feb. 2007.

[3] J. Clegg, A. C. Marvin, J. F. Dawson, and S. J. Porter, "Optimization of stirrer designs in a reverberation chamber," IEEE Trans. Electromagn. Compat., vol. 47, no. 4, pp. 824-832, Nov. 2005.

[4] P. Hallbjörner, "Estimating the number of independent samples in reverberation chamber measurements from sample differences," IEEE Trans. Electromagn. Compat., vol. 48, no. 2, pp. 354-358, May 2006.

[5] L. R. Arnaut, "Effect of local stir and spatial averaging on measurement and testing in mode-stuned and mode-stirred reverberation chambers," IEEE Trans. Electromagn. Compat., vol. 43, no. 3, pp. 305-325, Aug. 2001.

[6] O. Delangre, P. D. Doncker, M. Lienard, and P. Degauque, "Analytical angular correlation function in mode-stirred reverberation chamber," Electron. Lett., vol. 45, no. 2, pp. 90-91, 2009.

[7] F. Moglie and V. M. Primiani, "Analysis of the independent positions of reverberation chamber stirrers as a function of their operating conditions," IEEE Trans. Electromagn. Compat., vol. 53, no. 2, pp. 288-295, May 2011.

[8] C. Lemoine, P. Besnier, and M. Drissi, "Estimating the effective smaple size to select independent measurements in a reverberation chamber," IEEE Trans. Electromagn. Compat., vol. 50, no. 2, pp. 227-236, May 2008.

[9] X. Chen, "On independent platform sample number for reverberation chamber measurements," IEEE Trans. Electromagn. Compat., vol. 54, no. 6, pp. 1306-1309, Dec. 2012.

[10] R. J. Pirkl, K. A. Remley, and C. S. L. Patané, "Reverberation chamber measurement correlation," IEEE Trans. Electromagn. Compat., vol. 54, no. 3, pp. 533-544, Jun. 2012.

[11] G. Gradoni, V. Mariani Primiani, and F. Moglie, "Reverberation chamber as a multivariate process: FDTD evaluation of correlation matrix and independent positions," Prog. Electromagn. Res., vol. 133, pp. 217-234, 2013.

[12] X. Chen, "Using Akaike information criterion for selecting the field distribution in a reverberation chamber," IEEE Trans. Electromagn. Compat., to be published.

[13] G. Ferrara, M. Migliaccio, and A. Sorrentino, "Characterization of GSM non-line-of-sight propagation channels generated in a reverberating chamber by using bit error rates," IEEE Trans. Electromagn. Compat., vol. 49, no. 3, pp. 467-473, Aug. 2007.

[14] E. Genender, C. L. Holloway, K. A. Remley, J. M. Ladbury, G. Koepke, and H. Garbe, "Simulating the multipath channel with a reverberation chamber: Application to bit error rate measurements," IEEE Trans. Electromagn. Compat., vol. 52, no. 4, pp. 766-777, Nov. 2010.

[15] J. D. Sanchez-Heredia, J. F. Valenzuela-Valdes, A. M. MartinezGonzalez, and D. A. Sanchez-Hernandez, "Emulation of MIMO Rician fading environments with mode-stirred reverberation chambers," IEEE Trans. Antennas Propag., vol. 59, no. 2, pp. 654-660, Feb. 2011.

[16] P.-S. Kildal, X. Chen, C. Orlenius, M. Franzén, and C. Lötbäck Patané "Characterization of reverberation chambers for OTA measurements of wireless devices: Physical formulations of channel matrix and new uncertainty formula," IEEE Trans. Antennas Propagat., vol. 60, no. 8, pp. 38753891, Aug. 2012. 
[17] E. Jorswieck and H. Boche, "Majorization and matrix-monotone functions in wireless communications," Foundations Trends Commun. Inf. Theory, vol. 3, no. 6, pp. 553-701, 2006.

[18] N. A. Bagrov, "On the equivalent number of independent data," Tr. Gidrometeor. Cent., vol. 44, pp. 3-11, 1969.

[19] C. S. Bretherton, M. Widmann, V. P. Dymnikov, J. M. Wallace, and I. Bladé, "The effective number of spatial degrees of freedom of a timevarying field," J. Climate, vol. 12, no. 7, pp. 1990-2009, Jul. 1999.

[20] A. J. Laub, Matrix Analysis for Scientists and Engineers. Philadelphia, PA, USA: SIAM, 2005.

[21] X. Mestre, "Improved estimation of eigenvalues and eigenvectors of covariance matrices using their sample estimates," IEEE Trans. Inf. Theory, vol. 54, no. 11, pp. 5113-5129, Nov. 2008.

[22] X. Chen, P.-S. Kildal, and S.-H. Lai, "Estimation of average Rician Kfactor and average mode bandwidth in loaded reverberation chamber," IEEE Antennas Wireless Propag. Lett., vol. 10, pp. 1437-1440, 2011.

[23] C. L. Holloway, D. A. Hill, J. M. Ladbury, P. F. Wilson, G. Koepke, and J. Coder, "On the use of reverberation chamber to simulate a Rician radio environment for the testing of wireless devices," IEEE Trans. Antennas Propag., vol. 54, no. 11, pp. 3167-3177, Nov. 2006.

[24] C. Lemoine, E. Amador, and P. Besnier, "On the K-factor estimation for Rician channel simulated in reverberation chamber," IEEE Trans. Antennas Propag., vol. 59, no. 3, pp. 1003-1012, Mar. 2011.

[25] V. M. Primiani and F. Moglie, "Numerical simulation of reverberation chamber parameters affecting the received power statistics," IEEE Trans. Electromagn. Compat., vol. 54, no. 3, pp. 522-532, Jun. 2012.
[26] D. A. Hill, "Spatial correlation function for fields in a reverberation chamber," IEEE Trans. Electromagn. Compat., vol. 37, no. 1, pp. 138, Feb. 1995.

[27] A. Paulraj, R. Nabar, and D. Gore, Introduction to Space-Time Wireless Communication. Cambridge, U.K.: Cambridge Univ. Press, 2003.

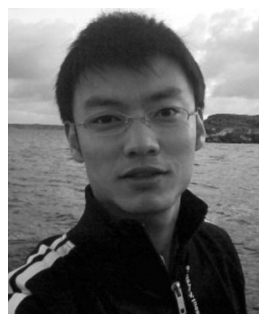

Xiaoming Chen received the B.Sc. degree from Northwestern Polytechnical University, Xi'an, China, in 2006, and the M.Sc. and Ph.D. degrees from the Chalmers University of Technology, Gothenburg, Sweden, in 2007 and 2012, respectively.

He is currently a Postdoctoral Research Associate in the Department of Signals and Systems, Chalmers University of Technology, Göteborg, Sweden. His current research interests include reverberation chamber measurements, multiantenna channel characterization, and statistical electromagnetics.

Dr. Chen is a Reviewer of the IEEE TRANSACTION ON ANTENNAS AND PROPAGATION, IEEE TRANSACTION ON ELECTROMAGNETIC COMPATIBILITY, IEEE TRANSACTION ON WIRELESS COMMUNICATIONS, and IEEE TRANSACTION ON VEHICULAR TECHNOLOGY. 